\title{
RG flow of AC Conductivity in Soft Wall Model of QCD
}

\author{
Neha Bhatnagar ${ }^{1, *}$ and Sanjay Siwach ${ }^{1, \dagger}$ \\ ${ }^{1}$ Department of Physics,Banaras Hindu University, \\ Varanasi-221005, India
}

\begin{abstract}
We study the Renormalization Group (RG) flow of AC conductivity in soft wall model of holographic QCD. We consider the charged black hole metric and the explicit form of AC conductivity is obtained at the cut-off surface. We plot the numerical solution of conductivity flow as a function of radial co-ordinate. The equation of gauge field is also considered and the numerical solution is obtained for $\mathrm{AC}$ conductivity as a function of frequency. The results for $\mathrm{AC}$ conductivity are also obtained for different values of chemical potential and Gauss-Bonnet couplings.
\end{abstract}

Keywords: Holographic QCD; Soft wall model; Transport Properties; Gauss-Bonnet coupling

\section{INTRODUCTION}

AdS/CFT correspondence [1] is widely used to study the dynamics of strongly correlated systems. AdS/CFT correspondence dictates that the dynamics of boundary theory can be studied by considering the fields in the dual gravity theory in the bulk AdS spacetime. A serious application of these ideas in QCD started from the work of Sakai and Sugimoto [2].

Alternatively, the phenomenological models of QCD [3], makes use of some known features of QCD and tried to incorporate these features in the bulk gravity theory. In these model the phenomena like chiral symmetry breaking are introduced by bifundamental fields dual to chiral condensate and confinement is realized by introducing an IR cut-off. This is further modified to include the Regge trajectories of mesons in the model and popularly known as soft wall model of QCD [4]. The role of IR cut-off is played by a dynamical wall in this model.

These models are studied further to investigate the phase structure and thermodynamics of QCD [5]. The transport properties like AC and DC conductivity and diffusion constant are also investigated in these models. [6-13]. Renormalization Group (RG) flow of transport co-efficients in theories dual to charged black hole is studied using membrane paradigm [14-22], where the dynamics of vector and tensor perturbations is used to study the flow equations for conductivity and diffusion constant. Here we adapt this formalism [14, 21, 22] to investigate the re-normalization flow of $\mathrm{AC}$ conductivity in the soft wall model of QCD. This is further generalized to include the effects of Gauss-Bonnet couplings in the bulk.

The paper is organized as follows. First, we consider the charged black hole solution of Einstein-Maxwell theory and perturbation equations for the bulk metric and gauge field in the soft wall model. We calculate the AC conductivity using the membrane paradigm and explicit results are given in the near horizon limit. We also plot

*Electronic address: bhtngr.neha@gmail.com

${ }^{\dagger}$ Electronic address: sksiwach@hotmail.com the full solution for AC conductivity as a function of frequency and the results in the probe limits are also plotted. Secondly, the results are obtained for AC conductivity while considering higher order gravity corrections in the action known as Gauss-Bonnet corrections. We also give explicit expression for DC conductivity at the cut off surface in the appendix.

\section{TRANSPORT COEFFICIENTS IN EINSTEIN-MAXWELL THEORY}

Let us consider the Einstein-Maxwell action in 5dimensions,

$$
S=\int d^{5} x \sqrt{-g} e^{-\phi}\left\{\frac{1}{2 \kappa^{2}}(R-2 \Lambda)-\frac{1}{4 g^{2}} F^{2}\right\},
$$

where $F^{2}=F_{m n} F^{m n}$ is the Lagrangian density of the Maxwell field, and $\phi$ is the dilaton field. The constant $\kappa^{2}$ is related to five dimensional Newton's constant $G_{5}$ as $\kappa^{2}=8 \pi G_{5}$, and the cosmological constant is related to AdS radius, $\Lambda=-6 / l^{2}$. The AdS/QCD correspondence relates five dimensional gravitational constant $2 \kappa^{2}$ and five dimensional gauge coupling to the rank of color group $\left(N_{c}\right)$ and number of flavours $\left(N_{f}\right)$ in the boundary theory, $\frac{1}{2 \kappa^{2}}=\frac{N_{c}^{2}}{8 \pi^{2}}, \quad \frac{1}{2 g^{2}}=\frac{N_{c} N_{f}}{4 \pi^{2}}$.

The charged black hole solution 21, 23, 24] of EinsteinMaxwell gravity in five dimensions with negative cosmological constant is given by,

$$
\begin{aligned}
d s^{2} & =\frac{r^{2}}{l^{2}}\left(-f(r) d t^{2}+\sum_{i=1}^{3} d x^{i} d x^{i}\right)+\frac{l^{2}}{r^{2} f(r)} d r^{2}, \\
A_{t} & =\mu\left(1-\frac{r_{+}^{2}}{r^{2}}\right)
\end{aligned}
$$

where $f(r)=1+a \frac{r_{+}^{6}}{r^{6}}-(1+a) \frac{r_{+}^{4}}{r^{4}}$, and the charge of the black hole is related with parameter ' $a$ ' and chemical potential $\mu$ as: $a=\frac{l^{2} \kappa^{2} Q^{2}}{6 g^{2}}, Q=\frac{2 \mu}{r_{+}}$. 
Defining, $u=\frac{r_{+}^{2}}{r^{2}}$ for simplification, the above metric can be written as,

$$
d s^{2}=\frac{r_{+}^{2}}{l^{2} u}\left(-f(u) d t^{2}+\sum_{i=1}^{3} d x^{i} d x^{i}\right)+\frac{l^{2} d u^{2}}{4 u^{2} f}
$$

where $f(u)=(1-u)\left(1+u-a u^{2}\right)$.

We take dilaton field in soft wall model as $\phi=c u$ and the constant $c=0.388 \mathrm{GeV}^{2}[4]$.

The equation of motion for the gauge field in the soft wall models can be written as,

$$
\frac{1}{\sqrt{-g}} \partial_{m}\left(\sqrt{-g} e^{-\phi} F^{m n}\right)=0 .
$$

Let us consider the metric and gauge field perturbations as,

$$
\begin{aligned}
g_{m n} & =g_{m n}^{0}+\tilde{h}_{m n} \\
A_{m} & =A_{m}^{0}+A_{m} .
\end{aligned}
$$

We scale the metric perturbation as $\tilde{h}_{m n}=e^{\phi} h_{m n}$ and take the Fourier decomposition of the fields as follows,

$$
\begin{aligned}
h_{m n}(t, z, u) & =\int \frac{d^{4} k}{(2 \pi)^{4}} e^{-i \omega t+i k z} h_{m n}(k, u) \\
A_{m}(t, z, u) & =\int \frac{d^{4} k}{(2 \pi)^{4}} e^{-i \omega t+i k z} A_{m}(k, u) .
\end{aligned}
$$

Now, focusing on the linearised theory for $h_{m n}$ and for vector field $A_{m}$ propagating in charged black hole background with the gauge condition $h_{u n}=0$ and $A_{u}=0$, one gets the equation of motion for vector modes of metric perturbations $h_{z}^{x}$ and $h_{t}^{x}$

$$
\begin{array}{r}
0=k f h_{z}^{\prime x}+\omega h_{t}^{\prime x}-3 a \omega u A_{x} \\
0=h_{t}^{\prime \prime x}-\frac{1}{u} h_{t}^{\prime x}-\frac{b^{2}}{u f}\left(\omega k h_{z}^{x}+k^{2} h_{t}^{x}\right)-3 a u A_{x}^{\prime} \\
0=h_{z}^{\prime \prime x}+\frac{\left(u^{-1} f\right)^{\prime}}{u^{-1} f} h_{z}^{\prime x}+\frac{b^{2}}{u f^{2}}\left(\omega^{2} h_{z}^{x}+\omega k h_{t}^{\prime x}\right)
\end{array}
$$

where, $b=\frac{l^{2}}{2 r_{+}}$. Similarly, the gauge field equation (4), becomes,

$$
0=A_{x}^{\prime \prime}+\left(\frac{f^{\prime}}{f}-c\right) A_{x}^{\prime}+\frac{b^{2}}{u f^{2}}\left(\omega^{2}-k^{2} f\right) A_{x}-e^{\phi} \frac{A_{t}^{\prime}}{f} h_{t}^{\prime x} .
$$

The gauge field equation is coupled with metric perturbations in charged black hole background and one has to resort to numerical methods to solve these equations in order to calculate the AC conductivity. However, in the near horizon regime an exact solution can be obtained.

We consider AC conductivity flow in the soft wall model in membrane paradigm [14] and using the fact that,

$$
\sigma_{A}(\omega, u)=\frac{J^{x}}{i \omega A_{x}}
$$

where the current density is given as,

$$
J^{x}=\frac{-1}{g^{2}} \sqrt{-g} e^{-\phi} F^{u x}+\frac{g_{x x}}{2 \kappa^{2}} \sqrt{-g} A_{t}^{\prime} h_{t}^{x} .
$$

Now, using equations (4), (13) and (14) the conductivity flow can be written as,

$$
\begin{aligned}
\frac{\partial_{u_{c}} \sigma_{A}}{i \omega}-\frac{g^{2} \sigma_{A}^{2}}{\sqrt{-g} e^{-\phi} g^{u u} g^{x x}} & -\frac{2 \kappa^{2} g^{x x} g^{4} \omega^{2}}{\sqrt{-g}} \frac{4 u^{3}\left(A_{t}^{\prime}\right)^{2}}{r_{+}^{2}} \\
& +\frac{\sqrt{-g} e^{-\phi} g^{x x} g^{t t}}{g^{2}}=0 .
\end{aligned}
$$
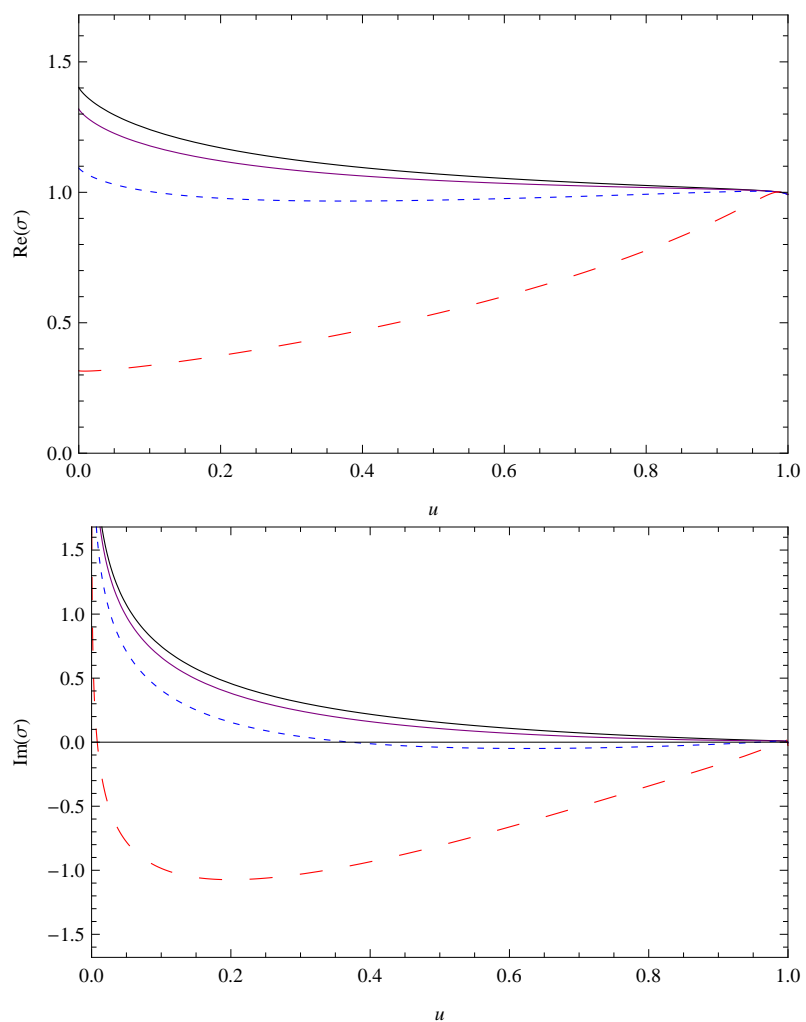

FIG. 1: The radial flow of $\mathrm{AC} \operatorname{conductivity}(\sigma)$ at fixed frequency $(\omega=0.999)$ with varying chemical potential $(\mu=0.01$ (black), 0.05(magenta), 0.1(dotted- blue), $0.25($ dashed red $))$

In the near horizon limit, $\sigma_{A}$ is a constant and can be evaluated by applying regularity condition at the horizon, $(\mathrm{u}=1)$

$$
\sigma_{A}(u=1)=\frac{e^{-c}}{g^{2}} \frac{r_{+}}{l}
$$

RG flow plots for AC conductivity has been shown in Fig.1. The frequency dependence of AC conductivity has been evaluated numerically and shown in Fig.3, Fig.4, Fig.5 and Fig.6. In the probe brane limit, taking $f(u)=$ $1-u^{2}$, the plots in Fig. 3, show striking similarity with condensed matter systems [25]. 


\section{TRANSPORT COEFFICIENTS WITH GAUSS BONNET CORRECTIONS}

We study the effect of Gauss-Bonnet(GB) coupling on the RG flow of conductivity in the soft wall model approach. The modified action with the GB term is given by,

$S=\int d^{5} x \sqrt{-g} e^{-\phi}\left\{\frac{1}{2 \kappa^{2}}\left(R-2 \Lambda+\alpha R_{G B}\right)-\frac{1}{4 g^{2}} F^{2}\right\}$,

where $R_{G B}=R^{2}-4 R_{M N} R^{M N}+R^{M N P Q} R_{M N P Q}$ is Gauss-Bonnet term and ' $\alpha$ ' is the Gauss Bonnet Coupling constant.

We consider the solution for Einstein-Maxwell-GaussBonnet(EMGB) system as 26 30].

$$
d s^{2}=\frac{r_{+}^{2}}{l^{2} u}\left(-f(u) N^{2} d t^{2}+\sum_{i=1}^{3} d x^{i} d x^{i}\right)+\frac{l^{2} d u^{2}}{4 u^{2} f},
$$

where

$$
\begin{aligned}
N^{2} & =\frac{1}{2}(\sqrt{1-4 \alpha}+1) \\
f(u) & =\frac{1}{2 \lambda}\left(1-\sqrt{1-4 \lambda(1-u)\left(1+u-a u^{2}\right)},\right.
\end{aligned}
$$

and $\lambda$ is related with the Gauss Bonnet coupling term as $\lambda=\frac{\alpha}{l^{2}}$.

Using the membrane paradigm as explained above for the charged black hole case and perturbation of metric and gauge fields as in previous section, the modified equations of motion for vector perturbations are given by,

$$
\begin{gathered}
0=\omega h_{t}^{\prime x}-\frac{u M^{\prime}}{M} f N^{2} k h_{z}^{\prime x}-\frac{3 a N^{2} A_{x} \omega}{M} \\
0=h_{t}^{\prime \prime x}+\frac{M^{\prime}}{M} h_{t}^{\prime x}+\frac{l^{4} b^{2}}{4 f} \frac{M^{\prime}}{M}\left(\omega k h_{z}^{x}+k^{2} h_{t}^{x}\right)-\frac{3 a N^{2} A_{x}^{\prime}}{M} \\
0=h_{z}^{\prime \prime x}-\frac{\frac{1}{u}-\frac{f^{\prime}}{f}+2 \lambda\left[\frac{f}{u}+u f^{\prime \prime}+u \frac{f^{\prime 2}}{f}-2 f^{\prime}\right]}{1+2 \lambda\left(u f^{\prime}-f\right)} h_{z}^{\prime x} \\
+\frac{l^{4} b^{2}}{4 N^{2} u f^{2}}\left(\omega^{2} h_{z}^{x}+\omega k h_{t}^{x}\right)
\end{gathered}
$$

$0=A_{x}^{\prime \prime}+\left(\frac{f^{\prime}}{f}-c\right) A_{x}^{\prime}+\frac{l^{4}}{4 N^{2} f^{2} r_{+}^{2} u}\left(\omega^{2}-k^{2} f\right) A_{x}-e^{\phi} \frac{1}{N^{2} f} h_{t}^{\prime x}$

where $M=\frac{1-2 \lambda f(u)}{u}$.

In order to determine the flow equation of $\mathrm{AC}$ conductivity, we consider the current density with the GB corrections defined as,

$$
J^{x}=-\frac{1}{g^{2}} \sqrt{-g} g^{u u} g^{x x} e^{-\phi} \partial_{u} A_{x}+\frac{1}{g^{2}} \sqrt{-g} \frac{4 u^{3}}{r_{+}^{2} N^{2}} A_{t}^{\prime} h_{t}^{x}
$$

The corresponding RG flow equation for AC conductivity (using definition equation (13)) becomes,

$$
\begin{array}{r}
\frac{\partial_{u_{c}} \sigma_{A}}{i \omega}-\frac{g^{2} \sigma_{A}^{2}}{\sqrt{-g} e^{-\phi} g^{u u} g^{x x}}-\frac{2 \kappa^{2} g^{x x} \sqrt{-g}}{g^{4} \omega^{2}}\left(A_{t}^{\prime}\right)^{2} \frac{4 u^{2}}{M r_{+}^{2}} \\
+\frac{\sqrt{-g} e^{-\phi} g^{x x} g^{t t}}{g^{2}}=0
\end{array}
$$

In the near horizon limit, $u_{c}=1$ (the cut-off horizon), we can get an exact expression for the AC conductivity,

$$
\sigma_{A}\left(u_{c}=1\right)=\frac{r_{+}}{g^{2}} \frac{e^{-c}}{l}
$$

$\mathrm{RG}$ flow plots for $\mathrm{AC}$ conductivity with $\mathrm{GB}$ corrections has been shown in Fig.3 and we can notice that the qualitative feature of the flow are similar to the case without Gauss Bonnet correction.
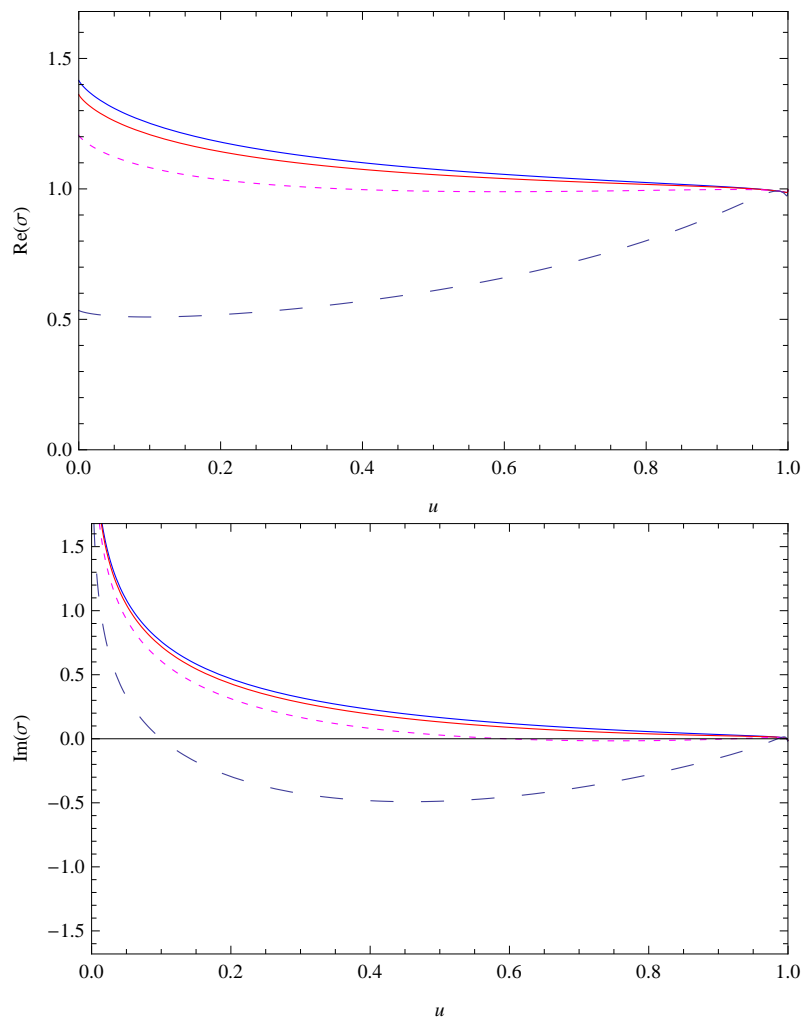

FIG. 2: The radial flow of AC conductivity $(\sigma)$ with GB $\operatorname{corrections}(\lambda=0.01)$ at fixed frequency $(\omega=0.999)$ with differnet $\mu=0.01$ (blue), 0.05 (Red), 0.1 (dashed magenta), 0.25 (dashed blue)

\section{CONCLUSIONS}

The soft wall model of Holographic QCD is used here to get the insights into the transport properties like 

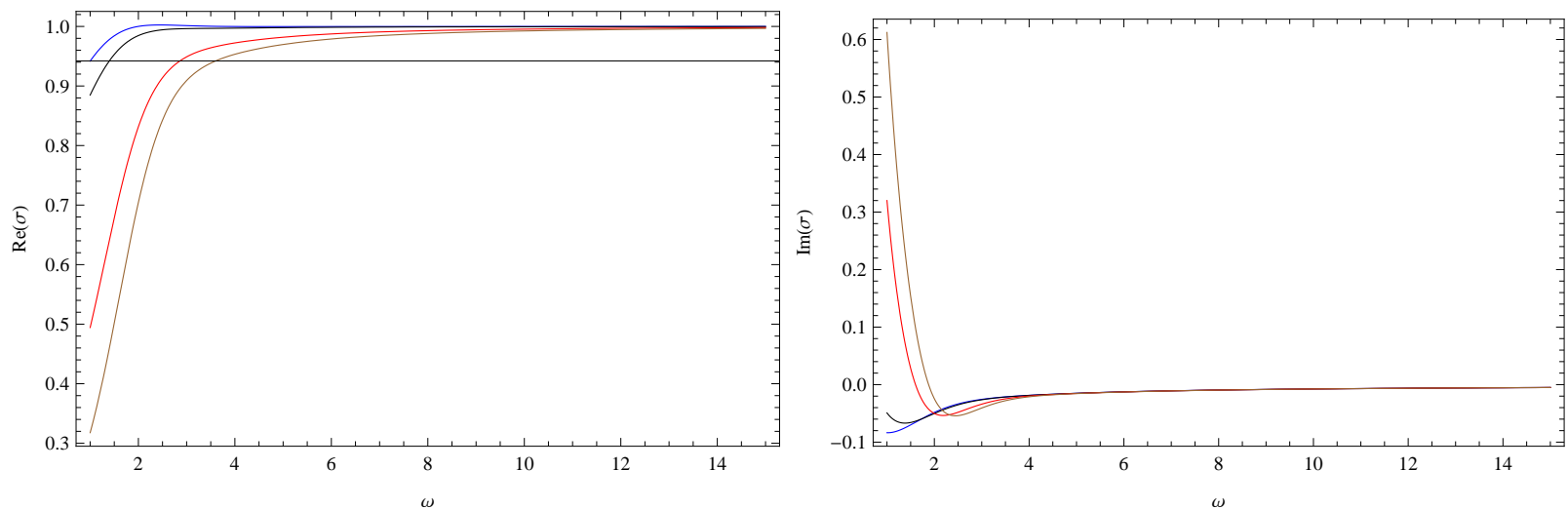

FIG. 3: Frequency dependence of $\mathrm{AC}$ conductivity $(\sigma)$ in the probe limit with varying chemical potential $(\mu=0.01($ blue $)$, 0.1(black), 0.9(red), 1.5(brown))

$\mathrm{AC}$ and DC conductivity in strongly coupled regime of QCD. The flow equations of $\mathrm{AC}$ and $\mathrm{DC}$ conductivity are considered for different values of chemical potential. The numerical solution of these equations enabled us to calculate the value of real and imaginary part of AC conductivity and the results seem to agree with the models, which consider the dynamics of condensate. This suggests that the soft wall model successfully captures the same features. This has also been noticed recently by 31] independently. In the probe limit our results (Fig.3) agree with existing results in the literature [21, 25, 32]. The results at high frequency (Fig.4, Fig.5) show oscillatory behavior of $\mathrm{AC}$ conductivity, which is reminiscent of Shubnikov de Haas effect. At low frequency, the Drude behavior [13] is observed (Fig.6). The GaussBonnet coupling does not change the results significantly.

\section{ACKNOWLEDGEMENT}

We acknowledge the financial support from the DST, Govt. of India, Young Scientist project.

\section{Appendix: DC CONDUCTIVITY}

In order to calculate DC conductivity, we consider the gauge field equation equation (12) in the limit $\omega, k=0$ the equation of motion for gauge-field becomes,

$$
A_{x}^{\prime \prime}+\left(\frac{f^{\prime}}{f}-c\right) A_{x}^{\prime}-e^{\phi} \frac{A_{t}^{\prime}}{f} h_{t}^{\prime x}=0
$$

The solution of $A_{x}(u)$, can be written as,

$$
A_{x}(u)=A_{x}(0)\left(1+\frac{3 a u\left(e^{c u}-1\right)}{c f^{\prime}-3 a u\left(e^{c u}-1\right)}\right),
$$

which can be used to calculate DC conductivity using the expression given in [14, 21]

$$
\sigma_{D C}=\left.\frac{\sqrt{-g}}{g^{2}} g^{x x} g^{u u} e^{-\phi} \sqrt{\frac{g_{u u}}{g_{t t}}}\right|_{u=1} \frac{A_{x}(1) A_{x}(1)}{A_{x}\left(u_{c}\right) A_{x}\left(u_{c}\right)} .
$$

Thus, we get the following expression for DC conductivity flow,

$\sigma_{D C}=e^{-c} \frac{r_{+}}{g^{2} l} \frac{(a-2)^{2}\left(a\left(-3 c u_{c}+3 e^{c u_{c}}+2 c-3\right)+2 c\right)^{2}}{\left(a\left(c-3 e^{c}+3\right)-2 c\right)^{2}\left(a\left(3 u_{c}-2\right)-2\right)^{2}}$

In the near horizon region, $u_{c}=1$, the DC conductivity takes the form,

$$
\sigma_{D C}=\frac{r_{+} e^{-c}}{g^{2} l}
$$

At the boundary $u_{c}=0, \mathrm{DC}$ flow is given as

$$
\sigma_{D C}=\frac{r_{+}}{g^{2} l} e^{-c} \frac{(a-2)^{2} c^{2}}{\left(a\left(c-3 e^{c}+3\right)-2 c\right)^{2}} .
$$

Following the above procedure, we can consider the DC conductivity with Gauss-Bonnet corrections. We obtain the identical expression for DC conductivity indicating that the flow is independent of Gauss-Bonnet terms.
[1] J. M. Maldacena, "The Large N limit of superconformal field theories and supergravity," Ad v. Theor. Math. Phys. 2, 231 (1998) hep-th/9711200, S. S. Gubser, I. R. Klebanov and A. M. Polyakov, "Gauge theory cor- relators from noncritical string theory," Phys. Lett. B 428, 105 (1998) hep-th/9802109, "Anti-de Sitter space and holography," Adv. Theor. Math. Phys. 2, 253 (1998) hep-th/9802150, 
[2] T. Sakai and S. Sugimoto, "Low energy hadron physics in holographic QCD," Prog. Theor. Phys. 113, 843 (2005) hep-th/0412141,

[3] J. Erlich, E. Katz, D. T. Son and M. A. Stephanov, "QCD and a holographic model of hadrons," Phys. Rev. Lett. 95, 261602 (2005) hep-ph/0501128,L. Da Rold and A. Pomarol, "Chiral symmetry breaking from five dimensional spaces," Nucl. Phys. B 721, 79 (2005) doi:10.1016/j.nuclphysb.2005.05.009 hep-ph/0501218.

[4] A. Karch, E. Katz, D. T. Son and M. A. Stephanov, "Linear confinement and AdS/QCD," Phys. Rev. D 74, 015005 (2006) hep-ph/0602229.

[5] S. Sachan and S. Siwach, "Thermodynamics of soft wall AdS/QCD at finite chemical potential," Mod. Phys. Lett. A 27, 1250163 (2012) doi:10.1142/S0217732312501635 arXiv:1109.5523 [hep-th]].

[6] D. T. Son and A. O. Starinets, "Minkowski space correlators in AdS / CFT correspondence: Recipe and applications," JHEP 0209, 042 (2002) hep-th/0205051. G. Policastro, D. T. Son and A. O. Starinets, "From AdS / CFT correspondence to hydrodynamics," JHEP 0209, 043 (2002) hep-th/0205052.

[7] P. Kovtun, D. T. Son and A. O. Starinets, "Holography and hydrodynamics: Diffusion on stretched horizons," JHEP 0310, 064 (2003) hep-th/0309213.

[8] J. Erlich, "How Well Does AdS/QCD Describe QCD?," Int. J. Mod. Phys. A 25, 411 (2010) arXiv:0908.0312 [hep-ph]].

[9] S. Jain, "Universal properties of thermal and electrical conductivity of gauge theory plasmas from holography," JHEP 1006, 023 (2010) arXiv:0912.2719 [hep-th]].

[10] K. -i. Kim, Y. Kim, S. Takeuchi and T. Tsukioka, "Quark Number Susceptibility with Finite Quark Mass in Holographic QCD," Prog. Theor. Phys. 126, 735 (2011) arXiv:1012.2667 [hep-ph]].

[11] M. Edalati, J. I. Jottar and R. G. Leigh, "Shear Modes, Criticality and Extremal Black Holes," JHEP 1004, 075 (2010) arXiv:1001.0779 [hep-th]].

[12] D. Albrecht, C. D. Carone and J. Erlich, "Deconstructing Superconductivity," Phys. Rev. D 86, 086005 (2012) doi:10.1103/PhysRevD.86.086005 arXiv:1208.2700 [hepth]].

[13] A. Donos and S. A. Hartnoll, "Interaction-driven localization in holography," Nature Phys. 9, 649 (2013) doi:10.1038/nphys2701 arXiv:1212.2998.

[14] N. Iqbal and H. Liu, "Universality of the hydrodynamic limit in AdS/CFT and the membrane paradigm," Phys. Rev. D 79, 025023 (2009) arXiv:0809.3808 [hep-th]].

[15] I. Heemskerk and J. Polchinski, "Holographic and Wilsonian Renormalization Groups," JHEP 1106, 031 (2011) arXiv:1010.1264 [hep-th]].

[16] Y. Matsuo, S. J. Sin, S. Takeuchi, T. Tsukioka and C. M. Yoo, "Sound Modes in Holographic Hydrodynamics for Charged AdS Black Hole," Nucl. Phys. B 820, 593 (2009) arXiv:0901.0610 [hep-th]].

[17] I. Bredberg, C. Keeler, V. Lysov and A. Strominger, "Wilsonian Approach to Fluid/Gravity Duality," JHEP 1103, 141 (2011) arXiv:1006.1902 [hep-th]].
[18] T. Faulkner, H. Liu and M. Rangamani, "Integrating out geometry: Holographic Wilsonian RG and the membrane paradigm," JHEP 1108, 051 (2011) arXiv:1010.4036 [hep-th]].

[19] S. -J. Sin and Y. Zhou, "Holographic Wilsonian RG Flow and Sliding Membrane Paradigm," JHEP 1105, 030 (2011) arXiv:1102.4477 [hep-th]].

[20] B. H. Lee, S. S. Pal and S. J. Sin, "RG flow of transport quantities," Int. J. Mod. Phys. A 27, 1250071 (2012) arXiv:1108.5577 [hep-th]].

[21] Y. Matsuo, S. -J. Sin and Y. Zhou, "Mixed RG Flows and Hydrodynamics at Finite Holographic Screen," JHEP 1201, 130 (2012) arXiv:1109.2698 [hep-th]].

[22] X. H. Ge, H. Q. Leng, L. Q. Fang and G. H. Yang, "Transport Coefficients for Holographic Hydrodynamics at Finite Energy Scale," Adv. High Energy Phys. 2014, 915312 (2014) arXiv:1408.4276 [hep-th]].

[23] A. Chamblin, R. Emparan, C. V. Johnson and R. C. Myers, "Charged AdS black holes and catastrophic holography," Phys. Rev. D 60, 064018 (1999) hep-th/9902170.

[24] X. H. Ge, Y. Matsuo, F. W. Shu, S. J. Sin and T. Tsukioka, "Density Dependence of Transport Coefficients from Holographic Hydrodynamics," Prog. Theor. Phys. 120, 833 (2008) arXiv:0806.4460 [hep-th]].

[25] S. A. Hartnoll, C. P. Herzog and G. T. Horowitz, "Building a Holographic Superconductor," Phys. Rev. Lett. 101, 031601 (2008) doi:10.1103/PhysRevLett.101.031601 arXiv:0803.3295 [hep-th]].

[26] R. G. Cai, "Gauss-Bonnet black holes in AdS spaces," Phys. Rev. D 65, $084014 \quad$ (2002) doi:10.1103/PhysRevD.65.084014 hep-th/0109133.

[27] R. G. Cai, Z. Y. Nie, N. Ohta and Y. W. Sun, "Shear Viscosity from Gauss-Bonnet Gravity with a Dilaton Coupling," Phys. Rev. D 79, 066004 (2009) doi:10.1103/PhysRevD.79.066004 arXiv:0901.1421 [hepth]].

[28] A. Buchel, J. Escobedo, R. C. Myers, M. F. Paulos, A. Sinha and M. Smolkin, "Holographic GB gravity in arbitrary dimensions," JHEP 1003, 111 (2010) doi:10.1007/JHEP03(2010)111 arXiv:0911.4257 [hep-th]].

[29] X. -H. Ge, Y. Ling, Y. Tian and X. -N. Wu, "Holographic RG Flows and Transport Coefficients in EinsteinGauss-Bonnet-Maxwell Theory," JHEP 1201, 117 (2012) arXiv:1112.0627 [hep-th]].

[30] Y. P. Hu, P. Sun and J. H. Zhang, "Hydrodynamics with conserved current via AdS/CFT correspondence in the Maxwell-Gauss-Bonnet gravity," Phys. Rev. D 83, 126003 (2011) doi:10.1103/PhysRevD.83.126003 arXiv:1103.3773 [hep-th]].

[31] S. S. Afonin and I. V. Pusenkov, "Soft wall model for a holographic superconductor," arXiv:1506.05381 [hep-th].

[32] S. A. Hartnoll, "Lectures on holographic methods for condensed matter physics," Class. Quant. Grav. 26, 224002 (2009) doi:10.1088/0264-9381/26/22/224002 arXiv:0903.3246 [hep-th]]. 

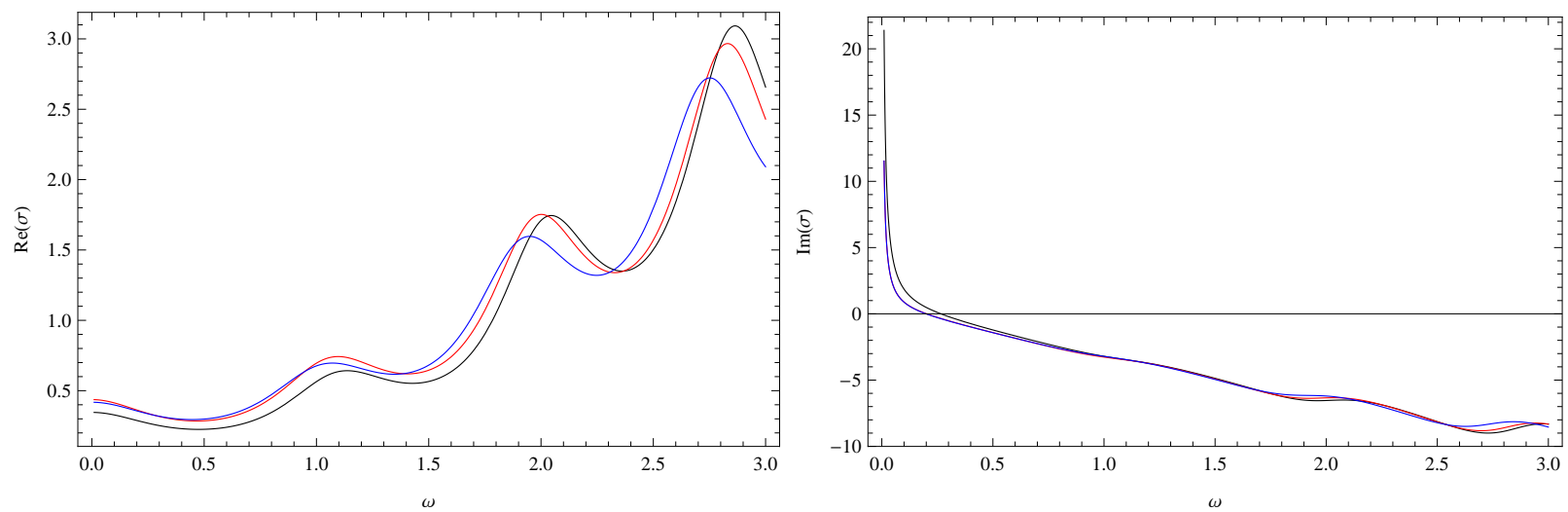

FIG. 4: Frequency dependence of AC conductivity $(\sigma)$ for fixed chemical potential ( $\mu=0.01)$ and varying Gauss-Bonnet terms $(\lambda=0$ (black), 0.01(red), 0.05(blue) $)$
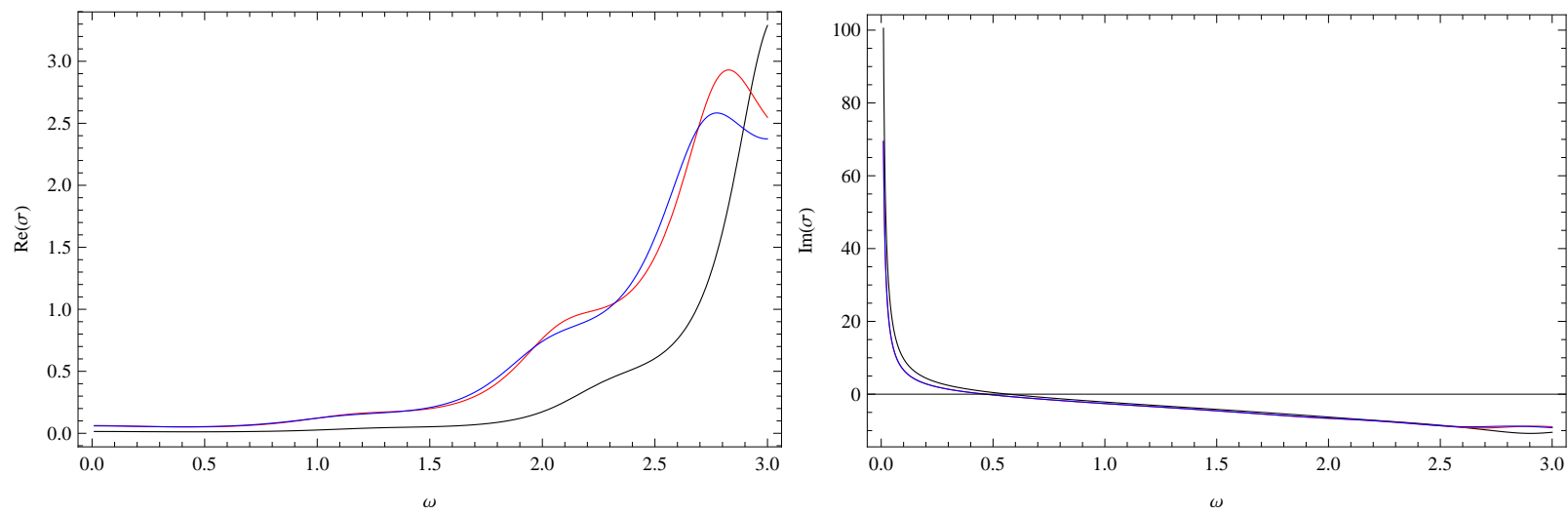

FIG. 5: Frequency dependence of AC conductivity $(\sigma)$ for higher chemical potential $(\mu=0.1)$ and varying Gauss-Bonnet term $(\lambda=0$ (black), 0.01(red), 0.05(blue)
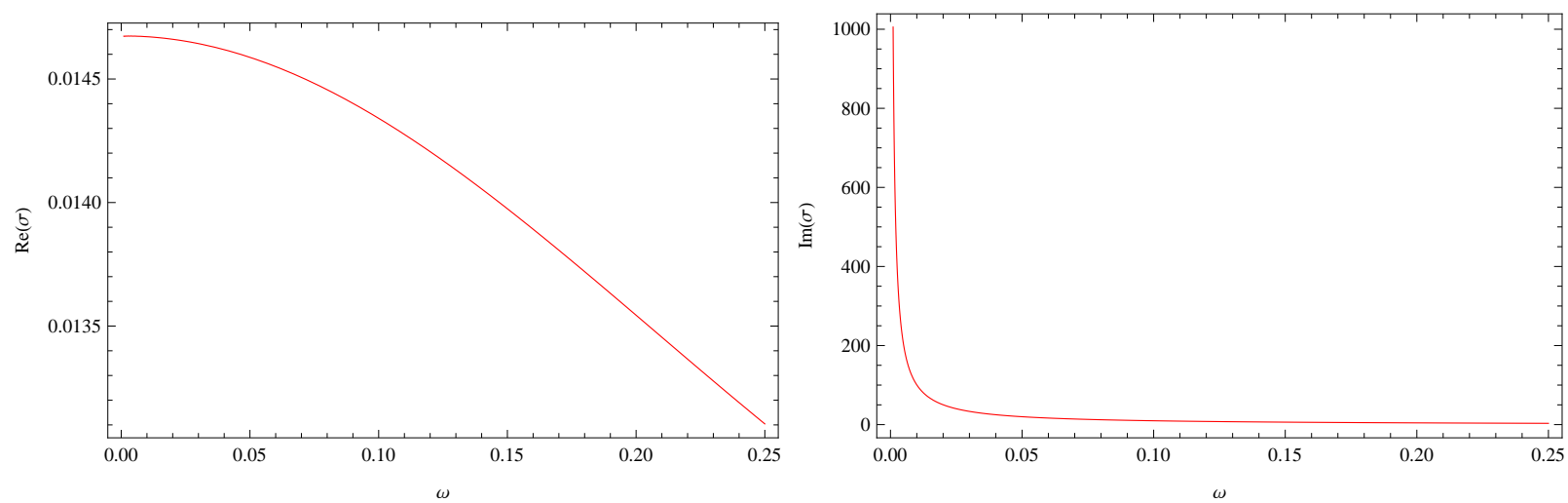

FIG. 6: Frequency(low) dependence of AC conductivity $(\sigma)$ for fixed chemical potential $(\mu=0.1)$ 